\title{
The dual role of Parylene $C$ in chemical sensing: acting as an encapsulant and as a sensing membrane for $\mathrm{pH}$ monitoring applications. \\ Tatiana Trantidou ${ }^{\mathrm{a}, *}$, David J. Payne ${ }^{\mathrm{b}}$, Vasileios Tsiligkiridis ${ }^{\mathrm{a}}$, Yu-Chun Chang ${ }^{\mathrm{a}}$, Christofer Toumazou ${ }^{\mathrm{a}}$, and Themistoklis Prodromakis ${ }^{\mathrm{a}, \mathrm{c}}$ \\ ${ }^{a}$ Centre for Bio-Inspired Technology, Department of Electrical and Electronic \\ Engineering, Imperial College London, London SW7 2AZ, UK \\ ${ }^{b}$ Department of Materials, Imperial College London, SW7 2AZ, UK \\ ${ }^{c}$ Nano Research group, Department of Electronics and Computer Science, University of Southampton, SO17 1BJ, UK \\ Email: tatiana.trantidou09@imperial.ac.uk, Tel: +44 (0)20 75940840.
}

\section{ABSTRACT}

In this work, we demonstrate a new property of Parylene $\mathrm{C}$ emphasizing on its application in $\mathrm{pH}$ sensing technologies. For many decades the material has been extensively used as a biocompatible inert encapsulant of implantable micro-devices. Towards a new understanding of the material's potential, we explore the transformation of Parylene $\mathrm{C}$ from a passive encapsulation membrane into an active $\mathrm{H}^{+}$sensing membrane using discrete MOSFETs to evaluate its chemical sensing performance. We employ oxygen plasma treatment to functionalize Parylene's $\mathrm{H}^{+}$ sensing capacity and enhance the chemical sensitivity, drift rates, and reliability of the sensing devices. Moreover, we demonstrate a versatile technique that enables the deployment of the material both as an encapsulant and as a sensing membrane in a single platform, in order to benefit from distinguishable and consistent sensitivities, and low leakage currents during $\mathrm{pH}$ measurements. Our investigation reveals that the selective modification of Parylene's surface chemistry yields reliable $\mathrm{pH}$ sensing devices, ensuring the best combination of sensitivity $(16.3 \mathrm{mV} / \mathrm{pH})$ and leakage currents (6-10 nA) over a reasonably wide $\mathrm{pH}$ range (4-10), while drift rates remain in low levels $(2.5-20 \mathrm{mV} / \mathrm{h})$. We believe that this study opens up new application horizons for Parylene, which is a new promising material in the emerging field of flexible electronics able to deliver low film thicknesses and high biocompatibility, while facilitating the application of mechanical stimulus.

Keywords: Parylene C, chemical sensing membrane, $\mathrm{pH}$ monitoring, oxygen plasma, extended gate, discrete MOSFETs

\section{Introduction}

Over the past few decades, poly(chloro-para-xylynene) (Parylene C) has been extensively used as a biocompatible encapsulant (barrier medium) of implantable microdevices, such as pacemakers [1] and catheters [2], chemical sensors [3,4], probes [5] and microelectrodes [6]. Parylene's excellent encapsulation properties have been attributed to its pinhole free nature above $100 \mathrm{~nm}$ [7], acting as an electrolyte- 
barrier layer, but also to its hydrophobic surface, resulting in the retraction of electrolytes that come in contact. Besides its numerous encapsulation applications, Parylene has been also employed together with Ion-Sensitive Field Effect Transistors (ISFETs) to suppress their $\mathrm{pH}$ sensitivity and serve as solid state reference electrodes, namely Reference Field-Effect-Transistors (REFETs), for differential ISFET/REFET $\mathrm{pH}$ measurements. The concept of the Parylene REFET has been introduced by Matsuo [8], who attempted to deposit a thin film of Parylene on a $\mathrm{Si}_{3} \mathrm{~N}_{4}$-gate ISFET in order to render it chemically inert. Nonetheless, the $\mathrm{pH}$ response of the Parylenegate ISFET showed a maximum sensitivity of $28 \mathrm{mV} / \mathrm{pH}$ below $\mathrm{pH} 4$, whereas for larger $\mathrm{pH}$ values the sensor was ion insensitive. Chemical modification of Parylene's surface with crown ether compounds was also implemented to examine the $\mathrm{K}^{+}$ sensitivity of the REFET, nevertheless, problems such as decreasing ionic sensitivity response over time and large drift were reported.

The electrostatic coupling between the ions and the gate insulating membrane has been the fundamental functional principle for a variety of well-established $\mathrm{H}^{+}$sensing technologies. This principle is mathematically expressed by the equation of electrostatics derived from Coulomb's law stating that the magnitude of the electric field created by a distribution of charges is inversely proportional to the dielectric thickness. This has been also proven by our recent work which demonstrated that thinner passivations result in an increase of the electrostatic coupling of $\mathrm{H}^{+}$and, therefore, in an improved sensitivity of the ISFET sensors [9]. This functional property has also been exploited for a different reason; to improve the sensors' performance, reliability and lifetime by having the active transducer in a remote location from the chemical sensing site, also known as the extended-gate approach [10,11]. A further deployment of this concept was recently demonstrated by our group, in which discrete Metal-Oxide-Semiconductor FETs (MOSFETs) were employed as the active transducers [12]. A set of devices with passive electrodes coated with an insulating membrane $\left(\mathrm{Si}_{\mathrm{x}} \mathrm{N}_{\mathrm{y}}\right.$ or $\left.\mathrm{TiO}_{2}\right)$ electrostatically couple the electrolyte ions and transduce the availing chemical potentials to the readout circuitry. The rest of the platform is encapsulated with Parylene $C$ to serve as a barrier between the metal interconnects and the electrolyte providing low leakage currents.

The hydrophobic nature of Parylene $\mathrm{C}$ certainly has rendered the material extremely popular for encapsulation purposes, nevertheless, its high biocompatibility and mechanical robustness have made the idea of exploiting the material in other applications interestingly appealing. Many research efforts have been realized to alter the hydrophobic properties of Parylene $\mathrm{C}$ with particular focus on the biomedical applications. Oxygen plasma treatment has been previously employed to effectively modify the surface properties of such films $[13,14]$, with special emphasis on utilizing the material as a cell culture substrate $[15,16]$. In other studies, Parylene has been employed for patterning cells and proteins either by enhancing attachment of proteins after UV exposure [17], or as a peel-off stencil [18].

In a recent research work, we extrapolated correlations between oxygen plasma treatment parameters (power intensity, exposure time), hydrophilicity and etching 
rates, and demonstrated selective hydrophilic patterning for self-alignment applications [19]. We also established that Parylene tends to partially restore its initial hydrophobicity within one week after treatment independently of the treatment conditions, nevertheless, restoration saturates to a certain point at which the material is still considered hydrophilic.

In this paper, we aim towards a new understanding of Parylene $\mathrm{C}$ properties beyond encapsulation, and investigate the material's potential in $\mathrm{H}^{+}$sensing. We employ $\mathrm{O}_{2}$ plasma treatment of Parylene in order to improve the chemical sensitivity, drift rates, and reliability of the sensors, and we propose the selective modification of the polymer's water surface affinity to utilize the material both as an encapsulant as well as a $\mathrm{pH}$ sensing membrane, by creating hydrophilic areas that are more sensitive to $\mathrm{H}^{+}$ions, while maintaining the desired interfaces hydrophobic to ensure low leakage currents during $\mathrm{pH}$ measurements. The work presented here demonstrates a new functional approach for Parylene $\mathrm{C}$, which can be employed both for in vitro but also for in vivo chemical monitoring applications.

\section{Materials and methods}

\subsection{Sensors fabrication}

PCB-based platforms (Fig. 1a) consisting of passive $\mathrm{Au}$ electrodes were thoroughly degreased in Acetone, Isopropanol (IPA) and Deionized (DI) water, and dehydrated for $60 \mathrm{~s}$ at $90{ }^{\circ} \mathrm{C}$. Parylene C films were deposited by Chemical Vapor Deposition, using a commercially available coater (PDS2010) by vaporizing $\left(150{ }^{\circ} \mathrm{C}\right)$ and then pyrolizing Parylene $\mathrm{C}$ dimer $\left(690^{\circ} \mathrm{C}\right)$, facilitating three distinct thicknesses (540 nm, $1 \mu \mathrm{m}, 3 \mu \mathrm{m}$ ) (Fig. 1b). This set of samples was used for evaluating $\mathrm{pH}$ sensitivity of hydrophobic Parylene C. A second group $(1 \mu \mathrm{m}, 3 \mu \mathrm{m})$ was rendered hydrophilic through oxygen plasma treatment at $400 \mathrm{~W}$ using an ultra high purity plasma etcher (Nano UHP) at a working pressure of 0.8 mbar. Two distinct film thicknesses were derived from the $1 \mu \mathrm{m}$-thick sensors depending on the exposure time-span; a) a residual thickness of $\sim 950 \mathrm{~nm}$, resulting from 1 min-treatment, and b) a thickness of $\sim 200 \mathrm{~nm}$ resulting from $10 \mathrm{~min}$-treatment. Film thickness was determined through calibration samples where Parylene $C$ was selectively removed to create a step that was measured by a Veeco Dektak Stylus profiler. A third group of 1 $\mu \mathrm{m}$-thick Parylene $\mathrm{C}$ platforms was rendered hydrophilic on top of the Au sensing sites only. The fabrication process included the patterning of Parylene $\mathrm{C}$ as follows: samples were cleaned and dehydrated as described above. Hexamethyldisilazane (HMDS) was spin-coated on the Parylene C coated PCBs, succeeded by a 1.4- $\mu$ mthick positive photoresist (AZ5214). The samples were then soft-baked on a hotplate at $90{ }^{\circ} \mathrm{C}$ for $60 \mathrm{~s}$, selectively exposed to UV light for $90 \mathrm{~s}$, and developed. Before inserted into the plasma etcher, the samples were prebaked at $110{ }^{\circ} \mathrm{C}$ for $60 \mathrm{~s}$ for hardening the protective photoresist mask. Oxygen plasma treatment was accomplished at $400 \mathrm{~W} / 10 \mathrm{~min}$, facilitating a film thickness of $200 \mathrm{~nm}$ on top of the electrode sites only, whereas hydrophobic Parylene remained intact $(1 \mu \mathrm{m})$ at the rest 
of the platform. For the fabrication of the patterned matrix the same procedure was followed with a different mask layout. For thicker Parylene films $(3 \mu \mathrm{m})$, photoresist AZ4562 (190 s UV exposure, 2 min development time) was utilized instead, due to its slower etching rate inside the plasma etcher. Plasma treatment was conducted at 400 W/35 min resulting in a $200 \mathrm{~nm}$ Parylene thickness on top of the metal sensing sites. After $\mathrm{O}_{2}$ plasma exposure, the photoresist was removed by immersing the platforms into ACE, IPA and then DI water.

\subsection{Experimental setup}

Throughout this study, discrete MOSFETs were employed in order to evaluate the material's chemical behavior. An array of p-type MOSFETs was hosted on an instrumentation system previously designed [12] together with an appropriate multiplexing scheme, the necessary biasing circuitry comprising of dual op-amp source-drain followers, and universal interfacing ports (see Suppl. fig. 1). To facilitate the transmission of the chemical potentials from the front-end PCB platforms to the read-out circuitry, the fabricated platforms were remotely connected with the gates of the MOSFETs through a connector. The extended-gate approach was selected in our work to improve the sensors' performance, reliability and lifetime as stated above. Depending on the available ionic resources, the binding of $\mathrm{H}^{+}$to the membrane surface in the vicinity of the sensing sites modulates the gate capacitance that leads to a corresponding output (drain) voltage. This in turn changes the MOSFETs threshold voltage and allows straightforward correlations between $\mathrm{H}^{+}$concentration and output voltage. Experiments were performed using $20 \mathrm{ml}$ of commercial $\mathrm{pH}$ buffer solutions (HANNA Instruments) with $\mathrm{pH}$ values of 4 (HI-7004), 7 (HI-7007) and 10 (HI-7010). Each sensor was immersed in each electrolyte solution for $2 \mathrm{~h}$ for the drift experiments, and for $200 \mathrm{~s}$ for the sensitivity experiments in the following order: $\mathrm{pH}$ 7, 4, 7, 10 and 7. In each case a $\mathrm{Ag} / \mathrm{AgCl}$ electrode was also immersed in the solution to serve as the reference electrode. Before testing a new electrolyte, the sensors and the $\mathrm{Ag} / \mathrm{AgCl}$ electrode were rinsed with DI water to remove any residual chemical compounds and wiped carefully. All experiments were performed at room temperature inside a Faraday cage for minimizing noise.

\section{Results and discussion}

\subsection{Evaluation of hydrophobic Parylene $C$ as a pH sensing membrane}

Chemical sensitivity of the three hydrophobic Parylene membranes $(540 \mathrm{~nm}, 1$ $\mu \mathrm{m}$ and $3 \mu \mathrm{m}$ ) was evaluated in solutions of $\mathrm{pH} 4,7$ and 10 through the $I_{D}-V_{G S}$ curves of the MOSFETs as the $\mathrm{Ag} / \mathrm{AgCl}$ reference electrode was swept from -2 to 1 V. Additionally, the sensors' transient responses across distinct electrolyte solutions were assessed using the instrumentation platform hosting the active components and the readout circuits (see supplementary material). The output voltage measured is the drain voltage derived from the dual op-amp source-drain followers, employed for biasing the MOSFETs. Average sensitivities for all three sensor types are illustrated in Fig. 1c. For each of the three sensing platforms, measurements of the output voltage $(\mathrm{mV})$ over 6 sensing sites were acquired at $\mathrm{pH} \mathrm{4,7}$ and 10, and a line was fitted to the experimental data. The slope $(\mathrm{mV} / \mathrm{pH})$ of the fitted line gives the average 
sensitivity of each Parylene sensor. The sensors' encapsulation quality was also extrapolated through leakage current measurements conducted for $\mathrm{I}_{\mathrm{D}}=100 \mu \mathrm{A}$ and $\mathrm{V}_{\mathrm{DS}}=0.5 \mathrm{~V}$, which are the typical biasing values defining the operation region of the MOSFETs. In the case of the $3 \mu \mathrm{m}$ thick Parylene membranes, the MOSFETs did not switch during the voltage sweep, indicating that the material is insensitive at $I_{D}=100$ $\mu \mathrm{A}$. Leakage current measurements were conducted at $\mathrm{V}_{\mathrm{GS}}=-0.85 \mathrm{~V}$ instead, where the difference between the three curves is more distinct. The conclusions driven from these experiments indicate that Parylene $\mathrm{C}$ displays a distinguishable chemical sensitivity in small film thicknesses $(23.97 \mathrm{mV} / \mathrm{pH}$ for $540 \mathrm{~nm})$, however, the material's behavior is not always consistent especially during the transition from $\mathrm{pH} 7$ to $\mathrm{pH} 10 . \mathrm{H}^{+}$sensitivity becomes lower and inconsistent in larger thicknesses $(1 \mu \mathrm{m})$ and eventually the material preserves its inertness and encapsulation integrity in much higher thicknesses $(3 \mu \mathrm{m})$. As previously described by Grahame [20], the electrolyteinsulator-metal interface results in the formation of a double layer region. A smaller dielectric thickness brings the inner Helmholtz layer, i.e. the interfacing $\mathrm{H}^{+}$ions, closer to the metal surface, introducing a smaller capacitance, which in turn leads to a larger output voltage. Contrary to what is stated in the literature regarding Parylene's pinhole-free nature above $100 \mathrm{~nm}$ [7], our experimental data support a pinhole-free behavior of Parylene at film thicknesses above $1 \mu \mathrm{m}$, indicated by a significant decrease in the leakage current as the membrane thickness increases from $540 \mathrm{~nm}$ to 3 $\mu \mathrm{m}$. Eventually, a film thickness of $1 \mu \mathrm{m}$ seems to be a transitional state between pinhole and pinhole-free status, also supported by the large standard deviations observed in Fig. 1c.

(Figures 1(a)-(c) about here)

\subsection{Evaluation of oxygen plasma treated Parylene C sensors}

\subsubsection{Conformally $\mathrm{O}_{2}$ plasma treated Parylene $\mathrm{C}$}

In order to examine whether an induced hydrophilicity would enhance Parylene's $\mathrm{pH}$ sensitivity, two sets of $1 \mu \mathrm{m}$-thick Parylene sensors were treated with oxygen plasma at $400 \mathrm{~W}$ for 1 and $10 \mathrm{~min}$, resulting in film thicknesses of 950 and $200 \mathrm{~nm}$ respectively. Longer exposure to $\mathrm{O}_{2}$ plasma does not only lead to a smaller membrane thickness, but also to an increased hydrophilicity. According to our recent findings [19], the water surface affinity of $1 \mu \mathrm{m}$-thick untreated and $\mathrm{O}_{2}$ plasma treated Parylene C films (evaluated through static contact angle measurements) corresponds to average contact angles of $78.85^{\circ} \pm 1.2^{\circ}, 4.5^{\circ} \pm 0.8^{\circ}$ and less than $1^{\circ}$ for untreated films and films treated (400W) for 1 and 10 min respectively. The plasma treated sensors were tested 15 days after plasma exposure, so that their induced hydrophilicity stabilizes according to previous findings [19]. Fig. 2a presents the transient response of the two sensor types when tested with various electrolytes, while Fig. 2b reveals the $I_{D}-V_{G S}$ curves of the thin-membrane sensor. As anticipated, the response of the Parylene-coated sensors with a thicker membrane appears noisier and exhibits lower chemical sensitivity comparing to thinner films. To ensure that this observation derives primarily from the smaller residual thickness and not from the extent of the induced hydrophilicity, we conducted the same experiments (measurements performed over 6 sensing sites) with a $3 \mu$ m-thick Parylene $\mathrm{C}$ sensor that was $\mathrm{O}_{2}$ plasma treated under the same conditions (400 W/10 min), yielding a residual thickness of $\sim 2.1 \mu \mathrm{m}$, and confirmed that the sensor did not exhibit any $\mathrm{pH}$ sensitivity. It is noteworthy that the improved response of the material is a combination of the degree of the induced hydrophilicity, and therefore the number of the available $\mathrm{H}^{+}$ 
binding sites, and the residual thickness, which is a common feature to all sensing membranes due to Coulomb electrostatic coupling.

(Figures 2(a)-(b) about here)

\subsubsection{Selectively $\mathrm{O}_{2}$ plasma treated Parylene $\mathrm{C}$}

Recently, we demonstrated how to selectively alter the hydrophobic surface properties of Parylene $C$ through standard lithography and oxygen plasma treatment [19]. In this study, we employ this technique to selectively expose the Parylene membrane on top of the gold sensing sites to $\mathrm{O}_{2}$ plasma (400 W/10 min), and benefit from the enhanced $\mathrm{pH}$ sensitivity of the induced hydrophilicity of the Parylene film, while concurrently preserving good encapsulation integrity of the sensor. A schematic cross-section of the fabrication process is illustrated in Fig.3a, while Fig.3b captures the selective hydrophilic patterning of the Parylene film which is evaluated by rinsing the whole PCB platform with electrolyte solution. Selectively treated sensors disclose a highly reactive behavior when they come in contact with the solution for the first time (15 days after $\mathrm{O}_{2}$ plasma exposure). Interestingly, their sensing capability appears to stabilize after a few weeks (Fig. 3c). Fig. 3d depicts the $I_{D}-V_{G S}$ curves of selectively treated sensors tested 45 and 90 days after plasma exposure respectively, indicating that $\mathrm{H}^{+}$sensitivity is slightly lower in the long-term, nevertheless, it still persists. The induced hydrophilicity of oxygen plasma treated Parylene $\mathrm{C}$ has been previously attributed to the formation of free carboxyl groups that are prone to bind with hydrophilic molecules $[13,14]$. The reactions that take place between the hydrophilic groups result in a diminished number of free binding sites on the film surface. As previously showed [19], this phenomenon leads to a partial restoration of the initial Parylene $\mathrm{C}$ natural hydrophobicity. However, restoration saturates at a certain point within a week after initial exposure to solution, and the induced hydrophilicity is further sustained.

(Figures 3(a)-(d) about here)

\subsubsection{Comparative characterization of Parylene $\mathrm{C}$ sensing devices}

Fig. 4a summarizes the average $\mathrm{pH}$ sensitivities derived from 6 channels of the three $1 \mu \mathrm{m}$-thick Parylene sensors (untreated, conformally and selectively treated at $400 \mathrm{~W} / 10 \mathrm{~min})$. Conformally and selectively plasma treated sensors exhibit comparable sensitivities (15.5 and $16.3 \mathrm{mV} / \mathrm{pH}$ respectively), whereas hydrophobic Parylene demonstrates a rather low sensitivity of $7.5 \mathrm{mV} / \mathrm{pH}$ which is not always consistent indicated by the large standard deviations. The encapsulation properties of the Parylene $C$ sensors were quantified by leakage current measurements through the reference electrode at $\mathrm{I}_{\mathrm{D}}=100 \mu \mathrm{A}$ (Fig. $4 \mathrm{~b}$ ). The observed sensitivities in all cases are well below the Nerstian [21], nonetheless, the relevant differences between the various $\mathrm{pH}$ values are still distinguishable, while the leakage current in all cases remains at relatively low levels. It is also worth mentioning that the substantially extended remote-gate employed in this work significantly increases the floating-gate capacitance resulting in a voltage-scaling, which is demonstrated by a lower sensitivity in every case. Results driven from these experiments support that the selectively treated Parylene $\mathrm{C}$ sensor combines good encapsulation properties and sufficient $\mathrm{H}^{+}$sensitivity. The versatility of this method enables us to fabricate a variety of sensors with thicker Parylene membranes to increase the sensor's 
encapsulation quality, and selectively modify the surface hydrophobicity by increasing correspondingly the plasma exposure time to get the desired final thickness. For example, we fabricated a $3 \mu \mathrm{m}$-thick Parylene membrane, which was selectively plasma treated $(400 \mathrm{~W})$ for $35 \mathrm{~min}$ to obtain a membrane thickness of $\sim 200 \mathrm{~nm}$ on top of the sensing sites (for consistency with the previous experiments), while preserving the thick layer $(3 \mu \mathrm{m})$ of hydrophobic Parylene on top of the electrode tracks to provide high encapsulation quality. The sensor exhibits good sensitivity, while the measured leakage current remains in very low levels $(9.8 \mathrm{nA}$ for $\mathrm{pH} 4,6.2 \mathrm{nA}$ for $\mathrm{pH} \mathrm{7}$, and $6.1 \mathrm{nA}$ for $\mathrm{pH} \mathrm{10),} \mathrm{when} \mathrm{compared} \mathrm{against} \mathrm{other}$ encapsulants such as epoxies and SU-8 [22]. Previously attained experimental results on $\mathrm{Si}_{\mathrm{x}} \mathrm{N}_{\mathrm{y}}$ and $\mathrm{TiO}_{2}$ sensing membranes using the same evaluation methods employed in this work [12], demonstrated chemical sensitivities of 35 and $22 \mathrm{mV} / \mathrm{pH}$ respectively which are obviously improved comparing to Parylene; however, these sensing devices suffer from larger leakage currents. Coating these devices with a thick layer of Parylene $\mathrm{C}(5 \mu \mathrm{m})$ provided an improved encapsulation quality with the leakage current being in comparable levels to the results presented here.

The reliability of all Parylene sensors exhibiting $\mathrm{H}^{+}$sensitivity is estimated in Fig. $4 \mathrm{c}$ as the percentage of the experiments in which the sensors demonstrated nerstian sensitivity out of the total number of experiments. Although all four sensors are $\mathrm{H}^{+}$sensitive, the plasma treated devices reveal a more consistent chemical behavior.

(Figures 4(a)-(c) about here)

As a proof-of-concept for illustrating Parylene's capacity to act both as an encapsulant and as a $\mathrm{H}^{+}$sensing membrane, we fabricated a pattered matrix with the layout presented in Fig. 5a. In this arrangement Parylene was locally rendered hydrophilic on top of a group of sensing sites, leaving the other areas hydrophobic. The sensors were again tested 15 days after treatment for consistency with the results acquired from previous experiments. Data were initially calibrated at $\mathrm{pH} 7$ to remove any offset between the distinct sensing sites and compensate for drift (see supplementary material for calibration method). The main purpose of calibration is to maintain a uniform and relative performance for all sensing sites of the array. Fig. 5b illustrates the 3-D representation of the calibrated data obtained from an active area of the matrix consisting of 20 sensing sites immersed into distinct electrolytes. The hydrophilic sites display larger voltage outputs relatively to the hydrophobic ones in all cases, namely a higher sensitivity response to the $\mathrm{pH}$ change. However, due to the interaction between adjacent sensing sites, some areas exhibit lower or higher response than normally expected.

(Figures 5(a)-(b) about here)

\subsection{Chemical drift}

Drift experiments were conducted for the three $1 \mu \mathrm{m}$-thick Parylene sensors for a time period of $2 \mathrm{~h}$. The main source of drift is attributed to the chemical interaction between the ions and the membrane surface, which causes alterations on the membrane's chemical composition and thickness. This drift-pH relation is articulated best through an exponential model. Conformally and selectively plasma exposed (400 W/10 min) Parylene sensors demonstrate lower drift than untreated Parylene, exhibiting the largest drift rates in $\mathrm{pH} 4$ solution (22.07 and $20.19 \mathrm{mV} / \mathrm{h}$ respectively), 
whereas their drift rates for $\mathrm{pH} 7$ and 10 are lower than $10 \mathrm{mV} / \mathrm{h}$ (Table 1). The large $\mathrm{H}^{+}$concentration in $\mathrm{pH} 4$ solution is likely to initiate massive reactions between the ions and the free carboxyl groups present on the surface of treated Parylene, as stated previously $[13,14]$.

(Table 1 about here)

\section{$3.4 X$-ray photoelectron spectroscopy analysis}

To understand the effect of oxygen plasma treatment on the chemical sensing performance of Parylene C, we performed X-ray photoelectron spectroscopy (XPS) measurements on a series of 1- $\mu$ m-thick Parylene $C$ films deposited on glass substrates, to investigate the composition of the surface chemical bonds before and after treatment. The $\mathrm{C} 1 \mathrm{~s}$ region core-line spectrum of untreated Parylene $\mathrm{C}$ is shown in Fig. 6a. At first glance it is apparent that there are two components of the $\mathrm{C} 1 \mathrm{~s}$ core line (centered at $\sim 284.5 \mathrm{eV}$ ), and shake-up satellite structure, due to $\pi-\pi *$ excitations on the benzene ring (centered at $\sim 291.2 \mathrm{eV}$ ). Upon peak fitting it can be determined that the $\mathrm{C} 1 \mathrm{~s}$ core line consists of three components labeled (I, II and III), corresponding to the $-\mathrm{CH}_{2}$, aromatic carbon and $\mathrm{C}-\mathrm{Cl}$ chemical environments, at $284.2 \mathrm{eV}, 284.6 \mathrm{eV}$ and $285.5 \mathrm{eV}$ respectively. There is also a clear signal from the $\mathrm{Cl}$ $2 \mathrm{p}$ core level, and a small amount of oxygen (surface contaminant). Upon oxygen plasma treatment $(400 \mathrm{~W} / 1 \mathrm{~min})$ it is apparent that the films become significantly more oxidized, shown in Fig. 6b, with the $\mathrm{O} 1 \mathrm{~s}$ core level at $\sim 532.5 \mathrm{eV}$. The peak at $\sim 289 \mathrm{eV}$ is most likely from carboxyl groups $(\mathrm{C}=\mathrm{O})$, which also agrees well with previous studies $[13,14]$. The sample treated at $400 \mathrm{~W} / 10 \mathrm{~min}$ has been greatly functionalized and shows significant surface oxidation (including the possibility of pinholes in the film by a concurrent etching process). The presence of a majority of oxygen species at the Parylene surface explains the increased hydrophilicity, although to understand the oxidation mechanisms fully, this system needs further study by XPS.

(Figures 6(a)-(b) about here)

\section{Conclusion}

In this work, we demonstrate a new property of Parylene $C$ emphasizing on its application in sensing technologies. Since now the material has been primarily used either as a biocompatible encapsulant of implantable microdevices or as an ionblocking layer aiming to suppress chemical sensitivity of the REFET in the ISFET/REFET topology. Towards a new understanding of the material's potential, we explore the role of Parylene $\mathrm{C}$ as an active $\mathrm{pH}$ sensing membrane using discrete MOSFETs to evaluate its chemical performance. Clearly, other circuitry, such as instrumentation amplifiers, can also be employed as alternative transducing schemes. Contrary to what is stated in the literature, hydrophobic Parylene seems to exhibit good encapsulation integrity only above $1 \mu \mathrm{m}$ indicating a pinhole-free behavior, whereas below this film thickness the material displays $\mathrm{H}^{+}$sensitivity which gradually increases for lower film thicknesses, it is not though consistent. Oxygen plasma treatment of Parylene $\mathrm{C}$ films was employed to reliably enhance the polymer's $\mathrm{pH}$ sensing capacity. Plasma induced hydrophilicity of Parylene $\mathrm{C}$ films yielded significant improvement of the $\mathrm{H}^{+}$sensitivity over the untreated, hydrophobic films 
(15.5 mV/pH vs. $7.5 \mathrm{mV} / \mathrm{pH})$. In addition, we demonstrate a versatile technique to selectively modify the surface hydrophilicity on top of the sensing sites and increase Parylene's $\mathrm{H}^{+}$sensing ability $(16.3 \mathrm{mV} / \mathrm{pH})$, while maintaining good encapsulation integrity within the same sensing platform. The observed sensitivities in all cases appear relatively low, however, this is primarily attributed to the evaluation method employed in this study (extended-gate), which compromises the sensitivity over robustness. Taking into account Parylene's high biocompatibility with tissue and biomolecules, the work presented here paves the way for potential application in 2-D toxicology assays, in which local immobilization of cells and biomolecules could enable the comprehensive interrogation of the cellular metabolism through appropriate membrane functionalization. Alternatively, since Parylene can deliver free-standing ultrathin (down to $1 \mu \mathrm{m}$ ) flexible films, in vivo application is also feasible based on a more hybrid approach of fabricated "bio-tattoos" performing simultaneously electrical and chemical measurements within the same recording platform. We believe that this study opens up new application horizons for Parylene, which is a new promising material in the emerging field of flexible electronics able to deliver low film thicknesses, high biocompatibility and facilitate the application of mechanical stimulus.

\section{Acknowledgments}

The authors would like to acknowledge the financial support of the A.G. Leventis Foundation.

\section{References}

[1] N. Iguchi, H. Kasanuki, N. Matsuda, M. Shoda, S. Ohnishi, S. Hosoda, Contact sensitivity to polychloroparaxylene-coated cardiac pacemaker, PACE 20 (1997) 372.

[2] L. Wolgemuth: Crystal-clear coating covers components, Med. Des. 6, 48-51 (2006) 331.

[3] T. Prodromakis, K. Michelakis, T. Zoumpoulidis, R. Dekker, C. Toumazou C, Biocompatible Encapsulation of CMOS based Chemical Sensor, IEEE Sensors conference (2009) 746.

[4] X. Liu, L. Li, B. Awate, R.M. Worden, G. Reguera, A.J. Mason, Biosensor Array Microsystem on a CMOS Amperometric Readout Chip, IEEE BioCAS (2011) 305.

[5] S. Takeuchi, D. Ziegler, Y. Yoshida, K. Mabuchi, T. Suzuki, Parylene flexible neural probes integrated with microfluidic channels, Lab on a Chip 5 (2005) 519.

[6] E.M. Schmidt, M.J. Bak, P. Christensen, Laser exposure of Parylene-C insulated microelectrodes, J. Neurosci. Meth. 62 (1995) 89.

[7] SCS Medical Coatings. Specialty Coating Systems, Indianapolis, Indiana. Available from: http://scscoatings.com/corporate/library.aspx (last accessed 18/4/2013).

[8] T. Matsuo, H. Nakajima, T. Osa, J. Anzai, Parylene-gate isfet and chemical modification of its surface with crown ether compounds, Sensors and Actuators 9 (1986) 115.

[9] T. Prodromakis, Y. Liu, T. Constandinou, P. Georgiou, C. Toumazou, Exploiting CMOS Technology to Enhance the Performance of ISFET Sensors, IEEE Electron Device Letters 31 (2010) 1053.

[10] L-T. Yin, J-C. Chou, W-Y. Chung, T-P. Sun, S-K. Hsiung, Separate structure extended gate H+-ion sensitive field effect transistor on a glass substrate, Sensors and Actuators B: Chemical 71 (2000) 106.

[11] C-G. Ahn, A. Kim, C.W. Park, C.S. Ah, J-H. Yang, T-Y. Kim, M. Jang, G.Y. Sung, Modified ion sensitive field effect transistor sensors having an extended gate on a thick dielectric, Appl. Phys. Lett. 96 (2010) 203702.

[12] T. Prodromakis, Y. Liu, J. Yang, D. Hollinghurst, C. Toumazou, A novel design approach for developing chemical sensing platforms using inexpensive technologies, IEEE BioCAS (2011) 369.

[13] J.W. Seong, K.W. Kim, Y.W. Beag, S.K. Koh, K.H. Yoon, J.H. Lee, Effects of ion bombardment with reactive gas environment on adhesion of Au films to Parylene C film, Thin Solid Films 476 (2005) 386.

[14] J.S. Song, S. Lee, S.H. Jung, G.C. Cha, M.S. Mun, Improved biocompatibility of parylene-C films prepared by chemical vapour deposition and the subsequent plasma treatment, J. Appl. Polym. Sci. 112 (2008) 3677. 
[15] T.Y. Chang, V.G. Yadav, S. De Leo, et al., Cell and protein compatibility of parylene-C surfaces, Langmuir 23 (2007) 11718.

[16] T. Hoshino, I. Saito, R. Kometani, et al., Improvement of neuronal cell adhesiveness on parylene with oxygen plasma treatment, J. Biosci. Bioeng. 113 (2011) 395.

[17] E. Delivopoulos, A.F. Murray, J.C. Curtis, Effects of parylene-C photooxidation on serum-assisted glial and neuronal patterning, J. Biomed. Mater. Res. 94 (2010) 47.

[18] C.P. Tan, B.R. Cipriany, D.M. Lin, H.G. Craighead, Nanoscale resolution, multicomponent biomolecular arrays generated by aligned printing with parylene peel-off, NanoLetters 10 (2010) 719.

[19] T. Trantidou, T. Prodromakis, C. Toumazou, Oxygen plasma induced hydrophilicity of Parylene-C thin films, J. Appl. Surf. Sci. 261 (2012) 43.

[20] D.C. Grahame, The electrical double layer and the theory of electrocapillarity, Chem. Rev. 41 (1947) 441.

[21] L. Bousse, The chemical sensitivity of electrolyte/insulator/silicon structures-Fundamentals of ISFET operation, Ph.D. dissertation, Technische Hogeschool, Delft, the Netherlands, 1982.

[22] T. Prodromakis, P. Georgiou, T.G. Constandinou, et al., Batch Encapsulation Technique for CMOS based Chemical Sensors, IEEE BioCAS (2008) 321. 


\section{FIGURE CAPTIONS}

Fig. 1. (a) PCB-based prototype platform with the Au/Parylene sensing sites located in the centre. (b) Schematic cross section of the fabrication process of untreated Parylene $\mathrm{C}$ sensors. (c) Average sensitivity and corresponding measured leakage currents over 6 channels of untreated $540 \mathrm{~nm}, 1 \mu \mathrm{m}$ and $3 \mu \mathrm{m}$ thick Parylene $\mathrm{C}$ films.

Fig. 2. (a) Representative transient response (non-filtered, non-calibrated data) of a single sensing site across distinct electrolytes of conformally $\mathrm{O}_{2}$ plasma treated $(400 \mathrm{~W}) 1 \mu \mathrm{m}$-thick Parylene sensors exposed for 10 (top) and 1 (bottom) min. (b) Corresponding $\mathrm{I}_{\mathrm{D}}-\mathrm{V}_{\mathrm{GS}}$ curves of conformally $\mathrm{O}_{2}$ plasma treated (400 W/10 min) sensor. A schematic cross section of the fabrication process is also included.

Fig. 3. (a) Cross-section of fabrication process of selectively $\mathrm{O}_{2}$ plasma treated Parylene $\mathrm{C}$ sensors. (b) Microscope image of single sensing site rinsed with electrolyte solution to evaluate selective hydrophilicity. (c) Transient response (non-filtered, non-calibrated data) across distinct electrolytes of selectively $\mathrm{O}_{2}$ plasma treated (400 W/10 min) $1 \mu \mathrm{m}$-thick Parylene sensor 15 (top) and 45 (bottom) days after treatment. (d) Corresponding $\mathrm{I}_{\mathrm{D}^{-}}$ $\mathrm{V}_{\mathrm{GS}}$ curves of selectively $\mathrm{O}_{2}$ plasma treated $(400 \mathrm{~W} / 10 \mathrm{~min}$ ) sensor 45 days and 90 days (subfigure) after treatment.

Fig. 4. (a) Chemical sensitivity evaluation of the $1 \mu$ m-thick Parylene devices over 6 channels and (b) corresponding average measured leakage currents. (c) Reliability of Parylene sensors indicated as the number of experiments in which the sensors exhibited nerstian sensitivity out of the total number of experiments conducted (measurements performed over $n$ sensing sites).

Fig. 5. (a) Layout of $1 \mu \mathrm{m}$-thick Parylene matrix: hydrophilic areas are indicated by the red circles. (b) Corresponding 3-D representation of voltage outputs indicating the response of the 20 sensing sites to distinct $\mathrm{pH}$ values.

Fig. 6. (a) $\mathrm{Al} \mathrm{K \alpha}$ core level photoemission spectrum of Parylene $\mathrm{C}$ in the $\mathrm{C} 1 \mathrm{~s}$ region. (b) Survey (left column) and $\mathrm{C}$ 1s spectra (right column) of: a) untreated Parylene $\mathrm{C}$ and b) $\mathrm{O}_{2}$ treated Parylene C (400 W/1 min.). 


\section{Biographies}

Tatiana Trantidou received her M.Eng. degree in Electrical \& Computer Engineering in 2009 from the Aristotle University of Thessaloniki, Greece and her M.Sc. degree in Biomedical engineering from Imperial College London, UK in 2010. She is currently pursuing a $\mathrm{PhD}$ in bio-inspired devices under the supervision of $\mathrm{Dr} \mathrm{T}$. Prodromakis and Prof. C. Toumazou at the department of Electrical \& Electronic Engineering at Imperial College London, UK. Her interests are in biomedical devices and point-of-care applications, while she already holds a patent in the field. Her area of research is currently being focused on the micro-fabrication of advanced cell culturing platforms with application in next-generation toxicology assays.

David J. Payne received his M.Chem. (2004) from Cardiff University and DPhil (2008), in experimental solidstate chemistry, from the University of Oxford, UK. From 2007 he was a Junior Research Fellow at Christ Church, University of Oxford. His research focuses on the spectroscopic determination of the electronic structure of oxide materials, and in particular developing (lab-based) near-ambient pressure x-ray photoelectron spectroscopy (NAPXPS). His interests are in oxide materials for energy applications including solar water splitting, photovoltaics and transparent conducting oxides. In 2011 he was appointed Lecturer in the Department of Materials at Imperial College London.

Vasileios Tsiligkiridis received the Diploma degree in Electrical and Computer Engineering in 2011 from the University of Patras, Greece and the M.Sc. degree in Biomedical Engineering in 2012 from Imperial College London, UK. His research interests include point-of-care medical diagnostics/monitoring solutions, biosensors and biomedical signal processing, with a particular interest in neuro applications.

Yu-Chun Chang received a Bachelor's degree in Mechatronics Engineering in 2010 from the University of Cape Town, South Africa. He obtained his M.Sc. in Control Systems Engineering in 2012 from Imperial College London, UK. He is currently pursuing a $\mathrm{PhD}$ degree in Neural \& Behavioral Sciences at the Max Planck Institute for Biological Cybernetics in cooperation with the University of Tübingen. His research interests include instrumentation, control systems and image processing for medical applications with a particular interest in magnetic resonance imaging.

Christofer Toumazou is a Professor of circuit design, Founder and Executive Director of the Institute of Biomedical Engineering at Imperial College London, UK. Prof. Toumazou has made outstanding contributions to the fields of low power analogue circuit design and current mode circuits and systems for biomedical and wireless applications. Through his extensive record of research, he has invented innovative electronic devices ranging from dual mode cellular phones to ultra-low power devices for both medical diagnosis and therapy. He has published over 320 research papers in the field of RF and low power electronics and is a member of many professional committees. He holds 23 patents in the field, many of which are now fully granted PCT. He is the founder of four technology based companies with applications spanning ultra low-power mobile technology and wireless vital sign monitors (Toumaz Technology Ltd., UK), biomedical devices (Applied Bionics PTE, Singapore), digital audio broadcasting (FutureWaves Pte Taiwan) and DNA detection (DNA Electronics Ltd., UK). These companies employ over $50 \mathrm{RF} / \mathrm{low}$ power engineers worldwide many of whom are Professor Toumazou's ex-graduate students. Professor Toumazou was invited to deliver the 2003 Royal Society Clifford Patterson Prize Lecture, entitled "The Bionic Man", for which he was awarded The Royal Society Clifford Patterson bronze medal. He was recently awarded the IEEE CAS Society Education Award for pioneering contributions to telecommunications and biomedical circuits and systems, and the Silver Medal from the Royal Academy of Engineering for his outstanding personal contributions to British engineering. In 2008, he was elected to the grade of Fellow of both the Royal Society and Royal Academy of Engineering.

Themistoklis Prodromakis is a Reader in Nanoelectronics within the Nano Research Group, ECS department at University of Southampton. He previously held a Corrigan Fellowship in Nanoscale Technology and Science, funded by the Corrigan Foundation and LSI Inc., within the Centre for Bio-inspired Technology at Imperial College London and a Lindemann Trust Visiting Fellowship in EECS UC Berkeley. Dr. Prodromakis is a member of the IEEE, the INE and the IET, and also serves as member of the BioCAS Technical Committee of the IEEE Circuits \& Systems Society. His background is in Electron Devices and micro/nano-electronics processing techniques, with his research being focused on bio-inspired devices for biomedical applications. 